\title{
Papierstau und Informationsfluss: Die Normierung der Bibliothekskopie ${ }^{1}$
}

\author{
von Monika Dommann
}

Auf der Weltausstellung von 1937 in Paris wurden die Besucher im Trocadero Zeugen einer amerikanischen „Expedition“. 2 Die Expedition galt nicht etwa entlegenen Ländern, sondern Beständen aus der Bibliothèque Nationale: alten Zeitungen aus der Revolutionsperiode und den kompletten Jahrgängen der Tageszeitung „Le Temps“. Der amerikanische Beitrag auf der Weltausstellung war von der Rockefeller Foundation finanziert. Herman Fussler, Bibliothekar der Bibliothek der University of Chicago, leitete das Unterfangen, begleitet von seiner Frau, die jeden Tag mit dem Taxi Zeitungen von der Nationalbibliothek in den Trocadero brachte. Unterstützt wurde er ferner von einem Studenten, einer Mikrofilmkamera, einem automatischen Entwicklungsgerät und Lesegeräten. Unter den Augen der Zuschauer wurden rund 200000 Seiten altes Papier auf Zellulose-Acetat verfilmt. Es ging um die Idee eines Freihandels mit wissenschaftlichen Ressourcen: „Speed free trade in ideas, as the railway and the steamship had speed trade in commodities". ${ }^{3}$ Die Negative kamen in die Obhut der American Library Association, Abzüge in die Bibliothek der University of Chicago und die Bibliothèque Nationale in Paris.

Siebzig Jahre später, im Jahr 2007, fährt Tag für Tag ein Lastwagen zur Cecil Green Library an der Stanford University und bringt Bücher an einen unbekannten Ort, wo sie gescannt und anschliessend in die riesige Datenbank von Google integriert werden. ${ }^{4}$ Zwischen der unter den Augen der Weltöffentlichkeit ausgeführten Kopierschau in Paris und der verborgenen Kopierfabrikation in Kalifornien liegen nicht bloss siebzig Jahre und ein Ozean, sondern auch mehrere technische Revolutionen (von der Photographie, über das Umkehrprisma, den Mikrofilm, den Xeroxkopierer bis zur Digitalisierung). Damit verknüpft ist auch die Zuspitzung eines Rechtskonfliktes um Urheberrechte, Droits d'auteur oder Copyrights, wie sie in den unterschiedlichen Rechtstraditionen genannt werden. Neben Büchern, Bibliotheka-

1 Der Text ist Teil eines grösseren Projektes, das vom Schweizerischen Nationalfonds finanziert wurde: Kopieren/Regulieren: Zur Kulturgeschichte von Vervielfältigungsapparaten, Werknormen und Verwertungsrechten. Ich danke Katja Girschik und Andrea Westermann, dafür, dass sie mich an der EASST Konferenz 2006 zu ihrem Panel „,The Multiplication of Things and its Consequences“ eingeladen haben und mit mir seither das Projekt einer „Geschichte der vielen Dinge“" weiterverfolgen.

2 M. Llewellyn Raney, The Decision of Paris, in: The Journal of Documentary Reproduction 1 (1938) H. 2, 151-161.

3 Ebd., 152.

4 Jeffrey Toobin, Googles's Moon Shot. The Quest for the Universal Library, in: The New Yorker, February 5 2007, 30-35. 
ren und Katalogen befindet sich mittlerweile in jeder Bibliothek eine grosse Anzahl von Photokopierern und Benutzerinnen und Benutzer besitzen neben zahlreichen Benutzerausweisen auch mindestens so viele Kopierkarten. Die Parallelbibliotheken wie JStor, Google Library, Universal Library, Open Content Alliance etc. sind mittlerweile grösser als die grössten Bibliotheken der Welt. Kopien auf Papier, Mikrofilm oder Speicherplatten gehören zu den viel benutzten Hilfsmitteln eines Wissenschaftlers. Sie haben das Lesen und die Herstellung, Speicherung und Diffusion von Wissen radikal verändert.

Der folgende Text untersucht die Voraussetzungen und Entstehungskontexte sowie die kulturellen, organisatorischen und rechtlichen Folgen des Kopierens in Bibliotheken im Zeitalter der Vervielfältigung seit dem 19. Jahrhundert. ${ }^{5}$ Welche Akteure und Techniken waren dabei involviert? Wie veränderten sich die sozialen Beziehungen und kulturelle Praktiken wie das Lagern, Suchen, Verteilen, Zählen und Ordnen und die damit verbundenen Rationalitäten und Normen? Es handelt sich dabei um ein breites Spektrum von Normierungspraktiken, die in diese Prozesse involviert sind: von technischen Standards, lokalen Reglementen und informellen Vereinbarungen, über die Rechtssprechung bis zu nationaler und internationaler Rechtsetzung. Schliesslich geht es dabei auch um die Frage, wie lokale Bibliotheken in eine ,globale“ Infrastruktur überführt wurden. ${ }^{6}$ Und nicht zuletzt handelt der Text von der Beziehung zwischen Europa und den USA, von der Rolle von Institutionen und Medien beim Transfer von Wissensressourcen und der Frage, wie und mit welchen Folgen Rechtsnormen in diesem komplexen Gefüge operieren. ${ }^{7}$

\section{Vom Abschreiben zum Abbilden}

Die Entstehung von neuen Kopiertechniken in der Welt des Lesens und Abschreibens ging zunächst mit einer Hinwendung zum „Original“ und zur Handschrift im Kontext der Professionalisierung und Institutionalisierung der Archiv- und Geschichtswissenschaften einher. Faksimilierungen (lat. „fac simile“), d.h. detailge-

5 Trotz ihrer prominenten Rolle als Arbeitstechnik für Wissenschaftler und für die Diffusion von wissenschaftlichen Texten haben Kopien bislang mit Ausnahme einiger punktueller Studien kaum wissenschaftliche Aufmerksamkeit auf sich gezogen: Wolfgang Ernst, (In)Differenz: Zur Ekstase der Originalität im Zeitalter der Fotokopie, in: Hans Ulrich Gumbrecht/Ludwik K. Pfeiffer (Hg.), Materialität der Kommunikation, Frankfurt a. M. ${ }^{2} 1995,498-518$. Hillel Schwartz, The Culture of the Copy. Striking Likeness, Unreasonable Facsimiles, New York 1996. Thorsten Lorenz, Copy!Right! Vom Gesetz der Kopie-Bildung, in: Rüdiger Campe/Michael Niehaus (Hg.), Gesetz. Ironie. Festschrift für Manfred Schneider, Heidelberg 2004, 85-107. Roger-Pol Droit, Fotokopierer, in: ders. (Hg.), Was Sachen mit uns machen. Philosophische Erfahrungen mit Alltagsdingen, Hamburg 2005, 151-153.

6 Zur Bedeutung von soziotechnischen Netzwerken für die Herstellung und Verbreitung von Universalität: Vgl. Bruno Latour, Wir sind nie modern gewesen. Versuch einer symmetrischen Anthropologie, Berlin 1995 [Paris 1991]. Zur Geschichte des Buches als Objekt des Wissens: Adrian Johns, Science and the Book in Modern Cultural Historiography, in: Studies in History and Philosophy of Science 29 (1997) H. 2, 167-194.

7 Vgl. dazu auch: Corynne McSherry, Who Owns Academic Work? Battling for Control of Intellectual Property, Cambridge/Mass. 2001. 
treue Nachdrucke von Handschriften und Urkunden existierten schon seit dem 17. Jahrhundert. ${ }^{8}$ Doch die Herausgeber von Faksimiles im 19. Jahrhundert wiesen auf die Andersartigkeit ihrer Projekte hin, die sie darin sahen, dass erstmals die Abbildung von Handschriften im Zentrum stand und die Begleittexte auf ein Minimum reduziert wurden. ${ }^{9}$ Diese Entwicklung ist kulturtechnisch folgenreich, handelt es sich doch um eine Verschiebung vom Abschreiben zum Abbilden. Die 1821 gegründete Ecole des Chartes in Paris, Kaderschmiede der Archivare und Historiker, begann in den 1830er Jahren mit den ersten Faksimilierungsprojekten. Während die Faksimiles des frühen 19. Jahrhunderts auf Pausen und lithographischen Verfahren basierten, entstanden in den 1850er Jahren erstmals Reproduktionen von Urkunden auf der Basis von photographischen Techniken. ${ }^{10}$ Die Faksimilierung mittels photographischer Verfahren zielte auf eine Verwissenschaftlichung historischer Methoden, wie sich aus Debatten um Originaltreue von Dokumenten und Quellen ablesen lässt. Die Anhänger der Photographie kritisierten dabei die Lithographien, die auf handwerklichen „Pausverfahren“ beruhten: „Ils ne pouvaient atteindre à une fidélité mécanique." 11 Das mechanische Verfahren der Photographie erschien ihnen dagegen objektiver. ${ }^{12}$

Die Anwendung der Photographie in Bibliotheken stiess anfangs auf bedeutende Widerstände, weil die technischen Anforderungen der Photographie (Licht, Wasser, Dunkelkammer) mit den konservatorischen Ansprüchen der Bibliotheken kollidierten. Nur wenige europäische Bibliotheken und Archive schufen spezielle photographische Einrichtungen. Die Bibliothèque Nationale in Paris baute 1877 einen Belichtungsraum mit Dunkelkammern und fliessendem Wasser in ihre Räumlichkeiten ein. ${ }^{13}$ Es wurden auch Bestände aus Provinzbibliotheken nach Paris gebracht; die Ausbeute waren etwa 20 Photographien von Handschriften und Druckschriften im Tag. Ein gleichzeitig erlassenes Reglement erklärte alle photographischen Aufnahmen in staatlichen Bibliotheken, Museen und Archiven für bewilligungspflichtig und gestattete sie nur unter Aufsicht eines Bibliotheksbeamten. Die Erlaubnis zur photographischen Reproduktion wurde vom Hinterlegen eines Negativs beim Ministerium und zwei Abzügen bei der entsprechenden Bibliothek abhängig gemacht. Diese Regelung stand in der Tradition des Pflichtexemplars für Druckerzeugnisse

8 Vgl. A. Giry, Manuel de diplomatique, [Paris 1893], ND Paris 1925, 41-50.

9 Vgl. Maurice Prou, L'êtat actuel des publications de fac-simile de chartes et autres documents d'archives, in: Actes du Congrès International pour la Reproduction des Manuscrits, des Monnaies et des Sceaux tenu à Liège, les 21, 22 et 23 août 1905, Bruxelles 1905, 62.

10 Vgl. die Dissertation von Daniela Saxer, Die Schärfung des Quellenblicks. Die geschichtswissenschaftliche Forschungspraxis in Wien und Zürich (1840-1914), Diss. Universität Zürich 2005, 337-395, welche auch das Faksimilierungsprojekt der „Monumenta graphica mediiaevi ex archivis et bibliothecis imperii Austriaci collecta" untersucht.

11 Prou, L'êtat, 63; Elie Berger, Les fac-similés photographiques et les calques, résumé de la polémique entre MM. de Pfugk-Harttung et Sybel, in: Bibliothèque de l'Ecole des Chartes XLVI (1885), 718-719.

12 Zum Konzept der mechanischen Objektivität: Lorraine Daston/Peter Galison, The Image of Objectivity, in: Representations 40 (1992), 81-128.

13 Emile Chatelain, La photographie dans les bibliothèques, in: Revue des bibliothèques 1 (1891), 224-241. 
bzw. des „dépôt légal“, deren älteste Bestimmungen aus dem Frankreich des 16. Jahrhunderts stammen und die Verleger zur Ablieferung von Pflichtexemplaren an die Bibliotheken verpflichteten. ${ }^{14}$ Photographische Aufnahmen waren auch in anderen europäischen Bibliotheken reglementiert oder von der Erlaubnis der Direktion abhängig. In einem Artikel in der „Revue des Bibliothèques“ aus dem Jahr 1891 kritisierte der Autor die „drakonischen Reglemente“, beispielsweise in der Bodleian Library in Oxford: „Ce qui étonne le plus, c'est les voir appliqués.“15 Auch die Vorschrift der Hinterlegung von teuren Negativen und Abzügen verurteilte er scharf: „Le dépot des clichés, auquel on a renoncé en France, est un mésure vexatoire et prohibitive“. ${ }^{16}$ Der Rückgriff auf das Negativ sei nicht mehr nötig, sobald die Aufnahme mittels Heliogravur oder Phototypie gedruckt sei. Die Idee des „dépôt légal“ war damit in Frage gestellt und die Diskussion darüber lanciert, ob die Bibliotheken auch für neue Medien ein adäquates Mittel der Diffusion seien, oder ob diese Rolle durch Reproduktion hinfällig werde.

Die Faksimilierungsprojekte im letzten Drittel des 19. Jahrhunderts avancierten „zum nationalstaatlich konzipierten und patriotisch aufgeladenen“17 Unterfangen und auch zum Geschäftsobjekt von Verlegern wie beispielsweise A. W. Sijthoff in Leiden, der ab 1897 Faksimiles griechischer und lateinischer Schriften verlegte. ${ }^{18}$ Der Diffusion dieser Kopien war jedoch angesichts der geringen Auflagen (150 Exemplare) ${ }^{19}$ und der „exorbitanten Preise“ ${ }^{20}$ Grenzen gesetzt. Um die Jahrhundertwende gelangten photographische Aufnahmen und Faksimilierungsprojekte in die Aufmerksamkeit internationaler bibliothekarischer Kreise. Diese sahen sie nun nicht mehr bloss als Quellen von Zerstörung, sondern sie entdeckten sie vielmehr als Mittel der Konservierung und Medium der Verbreitung kostbarer Materialien. Die Forderung nach einer internationalen Gesellschaft für die photographische Reproduktion von Handschriften und seltenen Drucken „ersten Ranges der Welt“ ${ }^{“ 21}$ wurde erstmals am internationalen Kongress der Bibliothekare in Chicago 1893 erhoben und 1905 wieder aufgegriffen. Damit verbunden war die Idee eines Weltkulturerbes, das auf Konservierung und Verbreitung von alten Hand- und Druckschriften beruhte. Die erste internationale Konferenz zur Frage der photographischen Reproduktion von Handschriften, Münzen und Siegeln in Liège im Jahr 1905 ist als Teil einer Blüte internationaler Konferenzen und Konventionen einzuordnen.

14 Heinrich Treplin, Das Bibliotheksrecht, in: Fritz Milkau (Hg.), Handbuch der Bibliothekswissenschaft, Leipzig 1933, Bd. 2: Bibliotheksverwaltung, 621-627, Robert Estivals, Le dépôt légal sous l'ancien régime de 1573 à 1791, Paris 1961.

15 Chatelain, Photographie, 230.

16 Ebd., 239.

17 Saxer, Schärfung, 358.

18 Paul Marc, Bibliothekswesen, in: K. W. Wolf-Czapek (Hg.), Angewandte Photographie in Wissenschaft und Technik, Berlin 1911, 59.

19 Karl Krumbacher, Die Photographie im Dienste der Geisteswissenschaften, in: Sonderabdruck: Neue Jahrbücher für das klassische Altertum 17 (1906), 613.

20 Marc, Bibliothekswesen, 60.

21 Paul Bermans, Les tentatives antérieures d'entente internationale pour la reproduction des manuscripts, in: Actes du Congrès International Liège, 44. 
Sie stand unter dem Schock des Brandes der Bibliothek in Turin im Jahr zuvor, der wertvolle Handschriften zerstörte: „Tous les bibliothèques finissent par brûler un jour.“22 Photographien wurden nun als ein Mittel gesehen, um Bibliotheken auf Zeitlosigkeit zu stellen. In Liège traf die alte Welt mit ihren grossen Bibliotheksschätzen auf Forderungen der neuen Welt nach Zugang zu den europäischen Beständen. Mills Gayley, ein Sprach- und Literaturwissenschaftsprofessor aus Berkeley, forderte die Gründung einer zentralen Kopier- und Verteilinstanz („Central clearing-house of research“23) in den USA zur Faksimilierung von Handschriften: „They are jealously guarded in the libraries of the Old World, and cannot be removed from them for love or money." 24 Gayleys Vision einer Tauschbeziehung zwischen Europa und den USA sah die zentrale Institution dabei in den USA, wo Faksimiles dringend benötigt würden und nicht in der alten Welt, wo zwar reiche Schätze an Handschriften schlummerten, aber das nötige Geld für deren Konservierung und Reproduktion nicht vorhanden sei. ${ }^{25}$ Er forderte freien Zugang zu Wissensbeständen $\mathrm{zu}$,liberalen“ und ,uneigennützigen“ Bedingungen. ${ }^{26}$ Seine Vision fusste auf einem Geschäftsmodell: Es sah ein Belegexemplar vor für die Bibliothek, welche das Dokument zur Verfügung stellt. Der Rest der Auflage würde auf Basis von Subskriptionsverträgen und individueller Nachfrage an Bibliotheken, Sammler und Universitäten verkauft. Mit rund 500000 Dollar Startkapital könnte ein „Corpus codicum universale" geschaffen werden, eine Parallelbibliothek aller Handschriften der Welt. Gayleys Skizze ist in der Sprache der Freihandelsbefürworter verfasst und basiert auf der Vorstellung der „economies of scale“: Hohe Investitionskosten sollten durch ein weltweites Netz von Bibliotheken und Sammlern getragen werden. Gayleys Modell einer zentralen Organisation der Vervielfältigung in den USA stiess bei europäischen Bibliotheken auf Skepsis und Ablehnung: „Il ne s'agit pas pour l'Europe de concéder un monopol à l'Amerique.“27

Inzwischen kündigte sich bereits eine neue Technik an, welche das Kopierverfahren verbilligte: Das Umkehrprisma. Abbé René Graffin, Mitbegründer der Patrologia Orientalis, präsentierte das Lichtprismaverfahren erstmals auf der Weltausstellung im Jahr 1900 in Paris. ${ }^{28}$ Es handelte sich um ein Verfahren um direkt auf photoempfindliches Papier (Bromsilberpapier) zu photographieren. Durch den Einsatz eines Spiegelprismas wurde die Schrift positiv gekehrt und erschien weiss auf schwarz. Damit war keine Dunkelkammer mehr nötig. Die Kosten für Aufnahmen verringerten sich um die Hälfte. ${ }^{29}$ Das Verfahren war im Kontext der Editionswis-

22 Actes du Congrès International Liège, 265.

23 Charles Mills Gayley, The Reproduction of Manuscripts from the American Point of View, in: Actes du Congrès International Liège, 214.

24 Gayley, Reproduction, 206.

25 Ebd., 209.

26 Ebd., 210.

27 Actes du Congrès International Liège, 303

28 Krumbacher, Photographie, 632.

29 Gewöhnliche Photographien kosteten 57-72 Pfennige bzw. 1,08 bis 1,33 Mark bei grösseren Formaten. Eine Prismenaufnahme kostete noch 25-30 Pf., bei Anstellung eines Photographen 37 Pf.: Ebd., 620. 
senschaften entstanden und kam auch zunächst in den Geisteswissenschaften zur Anwendung. Paul Marc unternahm im Auftrag des Byzantinisten Karl Krumbacher die „erste Expedition, bei der die Weissschwarzphotographie in grossem Umfang zur Anwendung kam“30: Während seiner Reise zum Berg Athos im Jahr 1906 machte er innerhalb von 22 Tagen 1307 Aufnahmen auf Bromsilberpapier und 102 Aufnahmen auf Planfilm, an einem Tag sogar bis zu 230 Aufnahmen. Verglichen mit den 20 Photographien in der Bibliothèque Nationale in den 1880er Jahren bedeute dies eine Zunahme um den Faktor zehn. Karl Krumbacher sah die Lichtprismenaufnahme als Technik der „objektiven Genauigkeit“31 und als Mittel der Konservierung des kulturellen „Erbes“ .32 Damit einher ging auch ein neuer Umgang mit Zeit: Kopien galten nun auch als Technik der „Beschleunigung der Arbeit“ ${ }^{\star 33}$. Die Vorbereitung für Editionsprojekte (Suchen, Reisen, Abschreiben) wurde nun als Zeitverschwendung angesehen, da sie „viele Jahre der besten Lebenszeit“ wegfressen würde. ${ }^{34}$ Das „,mechanische“35 Abschreiben von Hand verlor angesichts der Kopie die Eigenschaft eines gelehrten Handwerks.

Die Zerteilung von Büchern, der damit mögliche fragmentierte Zugriff und die Dekontextualisierung der Dokumente erforderte die Einübung einer Disziplin des Beschriftens der losen Photopapiere: „Es ist eine Selbsttäuschung zu glauben, dass man diese notwendigen Angaben später wirklich auf jeder Kopie nachtragen werde oder gar im Kopf behalten könne. Das mag früher möglich gewesen sein, als jedes Photogramm als etwas Besonderes angesehen wurde. "36 Kopien wurden zunächst wie Originale behandelt, es lag deshalb nahe, diese in die Bibliotheken zu integrieren. ${ }^{37}$ Die tradierte Norm der Hinterlegung von Negativen wurde durch die Technik jedoch in Frage gestellt: Da keine Negative mehr anfielen, konnten sie auch nicht hinterlegt werden. Wissenschaftler und Photographen kritisierten deshalb die Regel der Depositkopien, weil dadurch alle Aufnahmen mehrfach gemacht werden mussten, während andere Arten der Benutzung (manuelles Abschreiben oder Ausleihen) keine vergleichbare Gabe an die Bibliothek einschliessen würden. ${ }^{38}$ Zudem wurde in Frage gestellt, ob die Sammlung von Kopien für Bibliotheken überhaupt ein Gewinn sei und das alte Ordnungssystem der Bibliotheken nicht vielmehr inadäquat sei für die Lagerung und Distribution des neuen Mediums: Das Ordnen, Katalogisieren und Verwalten koste Zeit, Geld und Raum. ${ }^{39}$ Im Grunde lief die Argumentation auf den Gedanken hinaus, dass die Kopie die Funktion der Konservierung und Diffusion übernehme.

30 Ebd., 630.

31 Ebd., 620.

32 Ebd., 608.

33 Ebd., 619.

34 Ebd.

35 Marc, Bibliothekswesen, 63.

36 Ebd., 62.

37 Ebd., 76.

38 Ebd., 652.

39 Ebd., 652-653. 
Parallel zur Entwicklung der Prismaaufnahme entstand die Idee, die Mikrophotographie, mit der bereits in den 1850er Jahren experimentiert worden war, für Bibliotheken, genauer für die neu entstehende Dokumentationswissenschaft, nutzbar zu machen. ${ }^{40}$ Die „Bibliophotographie“ (oder „Bibliophote“), die später Mikrofiche und Mikrofilm hiess, wurde vom Institut International de la Bibliographie (IIB später IID) im Jahr 1906 erstmals als Speicher- und Diffusionsmedium von Büchern in die Diskussion gebracht. ${ }^{41}$ Sie beruhte auf einem zweistufigen Verfahren: In einem ersten Schritt sollten Dokumente auf einem photographischen Film verkleinert und in einem zweiten Schritt mittels eines Projektionsapparates rückvergrössert werden. „Le livre tend de nos jours à prendre la forme photographique“"42, hiess es programmatisch im Text von Robert Goldtschmit und Paul Otlet, dem Doyen der internationalen Dokumentationswissenschaft. ${ }^{43}$ Damit wurde die photographische Reproduktion von Druckschriften erstmals dezidiert als „Buch von morgen“44 bzw. „als neue Form des Buches“45 skizziert, weil sie nicht einfach ein Konservierungs- und Diffusionsmedium, sondern auch ein Substitut des Buches sei. Obwohl das Buch seit dem 15. Jahrhundert existiere, sei es nicht perfekt. Es verschwende Platz, sei teuer in der Produktion und deshalb ein Hindernis für die Wissensdiffusion: 60 Prozent der Bücher befänden sich in nur gerade zwölf Bibliotheken, wie sich überhaupt die Mehrzahl der Bücher in grossen Bibliotheken konzentriere. Als Alternative wurde die Idee eines mikrophotographischen Buches präsentiert. Als Medium waren Karteikarten von 12,5x7,5 cm mit 72 Bildern vorgesehen, die in Schubladen, ähnlich den bibliographischen Repertoires, aufbewahrt würden. Dieses neue Medium würde gemäss dem IIB künftig die Funktion der Bibliotheken verändern. Neben Konservation und Ausleihe müssten die Bibliotheken in der Reproduktion aktiv werden und sich damit der Verlagstätigkeit annähren: „Des copies fournies à volonté constituent en réalité un mode d'édition.“46 Man dachte an kleine Auflagen für Spezialistenzirkel und auch an Parallelpublikationen, an eine ,édition d'une œuvre sous une forme nouvelle, édition qui pourra être ent-

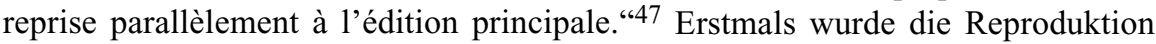
nicht mehr nur für rare Drucksachen und Manuskripte diskutiert, sondern für Aus-

40 Vgl. dazu: L'organisation de la documentation, in: Bulletin de L'Institut International de Bibliographie 13 (1908), 184-191; Paul Otlet, Traité de Documentation. Le livre sur le livre; Théorie et pratique, Bruxelles 1934.

41 Micro-Photography as Documentary Records, in: The British Journal of Photography, January 18 (1907), 39; Le livre microphotographique. Le Bibliophote ou livre à projection, in: Bulletin de L'Institut International de Bibliographie 16 (1911), 215-222.

42 Robert Goldschmidt/Paul Otlet, Sur une forme nouvelle du livre, in: Bulletin de L'Institut International de Bibliographie 12 (1907), 61-69.

$43 \mathrm{Zu}$ Paul Otlet: Françoise Levie, L'homme qui voulait classer le monde. Paul Otlet et le Mundaneum, Bruxelles 2006.

44 G. Vander Haeghen, Le Livre de Demain, in: Bulletin de L'Institut International de Bibliographie 12 (1907), 105-127.

45 Goldschmidt/Otlet, Sur une forme nouvelle, 44.

46 Le livre microphotographique, 220.

47 Ebd., 221. 
schnitte aus Büchern, Zeitungen und Zeitschriften. Das IIB entwarf das Konzept einer massgeschneiderten Lektüre angesichts der zunehmenden Arbeitsteilung in den Wissenschaften: „Il est donc de plus en plus nécessaire de permettre à chacun de se composer à volonté un livre de la question qui'il étude, par éxtrait de ces recueils.“ 48

\section{Vom Büchermagazin zum Verteilzentrum}

Die 1930er Jahre waren für die Entwicklung von Kopiertechniken in Bibliotheken ein Schlüsseljahrzehnt. Robert Goldschmidts und Paul Otlets Skizze einer komprimierten Bibliothek auf photographischem Film war nach dem Ersten Weltkrieg zur Marktreife gelangt, allerdings nicht für Bücher im Kontext der Bibliotheken, sondern für Checks im Kontext der Banken, wo der Kinematograph zu einem „Checkograph“ umgebaut wurde. ${ }^{49}$ Die Umkehrprisma- oder Photostatapparate, wie sie in den USA genannten wurden, funktionierten inzwischen weitgehend automatisiert und verwendeten nicht mehr gewöhnliches Photopapier, sondern ein Papier mit einer dünnen photographischen Schicht, das weniger zerbrechlich war und beschriftet werden konnte. ${ }^{50}$

Im deutschsprachigen Raum hiessen die Prismenaufnahmen bald nur noch Photokopien, benannt nach der gleichnamigen Firma „Photokopie GmbH“ (und ihren Apparaten, den Photokopist Apparaten), die 1927 gegründet wurde. ${ }^{51}$ Nachdem die Deutsche Bücherei in Leipzig bereits 1928 einen Lichtprismaapparat angeschafft hatte, ${ }^{52}$ eröffnete die Photokopie GmbH in der Staatsbibliothek in Berlin in einem Nebenraum des Lesesaals eine Filiale mit drei Apparaten (Photokopie und Mikrofilm), welche rund 200 bis 300 Aufnahmen im Tag herstellte. Ein Werbeprospekt präsentierte das „mechanisch abgeschriebene Buch“53 im Geist der Zeit als Rationalisierung des wissenschaftlichen Arbeitens, das jedes Studierzimmer zum Lesesaal umfunktioniere.

Im Gegensatz zum Photokopierapparat, der zu Beginn der 1930er Jahre mehr oder weniger technisch stabilisiert war, fing die Weiterentwicklung der Mikrofilmtechnik zu diesem Zeitpunkt erst an. Die Mikrokopie galt wegen den niedrigeren Produktions- und Transportkosten und dem geringeren Raumbedarf zur Archivie-

48 Ebd. Eine andere Technik der massgeschneiderten Lektüre ist der Zeitungsausschnitt: Anke te Heesen, Der Zeitungsausschnitt. Ein Papierobjekt der Moderne, Frankfurt a. M. 2006.

49 M. Llewellyn Raney, American Trends in Textual Reproduction, in: F. I. D. Communicationes 6 (1939), 77; Robert C. Binkley, Manual on Methods of Reproducing Research Materials. A Survey Made for the Joint Committee on Materials for Research of the Social Science Research Council and the American Council of Learned Societies, Ann Arbor 1936, 172.

50 Eine Übersicht über die verschiedenen Apparaturen: J.P.C. Van Asperen, Neuzeitliche photographische Reproduktionsverfahren, in: I.I.D. Communicationes 1 (1934) H. 3, 1-12 und 1 (1934) H. 4, 2-24.

51 Centralblatt für Bibliothekswesen 49 (1932), 312; Der Mikrofilm 2 (1952) H. 2, 1.

52 Heinrich Schreiber, Pflicht und Recht der Bibliotheksphotokopie, in: UFITA 7 (1934), 457 458.

53 Der Lesesaal von morgen... [Werbeschrift der Photo-Copie G. m. b. H], Berlin o. J. [1931], 5. 
rung im Vergleich zur Photokopie als Medium der Zukunft. Paul Otlet zeigte sich 1934 euphorisch: „L'économie est donc de 18 fois quant au poinds et de 96 fois quant au volume." 54 Der Direktor einer deutschen Bibliothek träumte von einer portablen Bibliothek: „Sie können also eine Bibliothek, die doppelt so gross ist, wie die grössten Bibliotheken der Welt, in eine Schachtel, die kaum grösser ist als eine Zigarrenkiste, mit auf die Reise nehmen." 55 Angesichts des „lawinenartigen Anwachsens“ und der „Massenbenutzung“ von Bibliotheken seit Mitte des 19. Jahrhunderts ${ }^{56}$ sahen Bibliothekare in der Miniaturisierung und der Ersetzung von Papier durch Film die Rettung. Die Bücher- und Papiermassen hatten in der Sprache der Bibliothekswissenschaften durchaus etwas Bedrohliches, das mit den Mitteln der Architektur, der Technik und der Administration kontrolliert werden musste. Die dabei verwendeten Metaphern aus dem Fundus des Wasserbaus und der Naturgewalten sind vergleichbar mit dem Sprachgebrauch im Zusammenhang mit Menschenmassen. ${ }^{57}$ Das Büchermagazin und die verstellbaren Regale waren eine erste architektonische Antwort auf das Wachstum der Bestände gewesen; Otlet sah im Mikrofilm die Lösung angesichts eines drohenden „Staus“ durch Papiermassen: „Emplacement réduit, évitant l'encombrement". 58

Es waren dann die amerikanischen Forschungsbibliotheken in Kooperation mit der Photoindustrie sowie staatlichen Organisationen, die finanziert durch Stiftungen seit Mitte der 1930er Jahre die vielen Experimentalanordnungen zum automatisierten bibliothekarischen Hilfsmittel weiterentwickelten und im grossen Massstab zur Anwendung brachten. ${ }^{59}$ Der erste Anwendungskontext waren grossangelegte Verfilmungsprojekte in den Bibliotheken Europas. Im British Museum installierte die Firma Edward Brothers aus Ann Arbor 1935 eine 35mm Kamera für die Verfilmung aller englischsprachigen Bücher vor $1550 .{ }^{60}$ Die Firma University Microfilms, die aus der Edward Brothers hervorging, betrieb diesen Service als kommerzielle Tätigkeit und bot auf Subskriptionsbasis von 500 \$ im Jahr (oder 0.5 Cent pro Seite) alte Drucke auf Mikrofilm an. ${ }^{61}$ Der öffentlichkeitswirksame Höhepunkt der Verfilmungsprojekte in Europa war der eingangs erwähnte US-amerikanische Beitrag

54 Paul Otlet, Le livre photomicrographique, in: I. I. D. Communicationes 1 (1934) H. 2, 19.

55 Walter Schürmeyer, Mitteilungen über einige technische Neuerungen und Anwendungsmethoden fotografischer Hilfsgeräte für das dokumentarische Arbeiten, in: Internationales Institut für Dokumentation (I.I.D.) (Hg.), Rapports Conference de documentation Copenhague 1935, Bruxelles 1935, Schü.1-Schü. 8 .

56 Milkau, Bibliotheksverwaltung, 36.

57 Elias Canetti, Masse und Macht, Frankfurt 1985 [ $\left.{ }^{1} 1960\right], 81-100$.

58 Otlet, Le livre photomicrographique, 19.

59 Vgl. Atherton Seidell, Film-strip Copies of Scientific Publications, in: I. I. D. Communicationes 2 (1935) H. 2, 24-27; Eugene B. Power, University Microfilms, in: Journal of Documentary Reproduction 2 (1939) H. 1, 21-28; The Present Status of Equipment and Supplies, in: Journal of Documentary Reproduction 1 (1938) H. 3, 4-56; Rockefeller Foundation, Annual Report, New York 1937-945.

60 I. I. D. Communicationes 3 (1936) H. 1, ZZ.2-ZZ.4.

61 Microfilm Abstracts, in: F. I. D. Communicationes 8 (1941), 20-21; Publication of Doctoral Theses by Microfilm, in: F. I. D. Communicationes 8 (1941), 53-54; Power, University Microfilms, 21-28. 
auf der Weltausstellung in Paris im Jahr 1937, der den Besuchern die Übertragung von papierenen Medien auf Zellulose-Acetat vorführte. Auch die Photoindustrie stieg in das Geschäft ein: „Recordak“, der Hersteller von Mikrofilmapparaten und Lesegeräten, sicherte sich beispielsweise mit einen Vertrag die Rechte für die Verfilmung und den Verkauf der „New York Times“ auf Mikrofilm. ${ }^{62}$ Der zweite Anwendungskontext waren Versuche, Dissertationen auf Mikrofilm statt in Buchform zu veröffentlichen, wobei wiederum die University Microfilms in Kooperation mit Universitäten eine Vorreiterrolle einnahm. Gegen eine Gebühr von 15 \$ wurden Dissertationen mittels ,abstracts“ in Zeitschriften angekündigt und dann ,on demand" publiziert. ${ }^{63}$ Ein drittes Verwendungsfeld für den Mikrofilm war die Herstellung von Mikrokopien von Zeitschriftenaufsätzen durch Bibliotheken für Wissenschaftler; eine Pionierrolle nahm der 1934 errichtete Bibliofilm Service ein. ${ }^{64}$ Es handelte sich dabei um einen Verbund diverser Forschungsbibliotheken in Washington DC auf einer Non-Profit Basis unter Ägide des American Documentation Institut. Die Dienstleistung stiess sofort auf rege Nachfrage: Die Aufträge für Zeitschriftenaufsätze stiegen von 470 pro Monat im November 1934 auf 6597 im Mai 1935.

In Frankreich gab es seit 1935 einen Mikrofilmapparat im Maison de la Chimie, ein weiterer in der Bibliothèque Nationale wurde der Bibliothek von der Rockefeller Foundation im Zusammenhang mit amerikanischen Nachfragen nach alten Drucken zur Verfügung gestellt. ${ }^{65}$ Nur amerikanischen Bibliotheken wie die Library of Congress ${ }^{66}$ mit Zugriff auf Stiftungsgelder (oder europäische Bibliotheken, welche für amerikanische Verfilmungsprojekte interessant waren) war es möglich, die Mitte der 1930er Jahre verfügbaren leistungsfähigen Apparate zu erwerben. Der Gründer von University Microfilms sah die amerikanische Dominanz in der Mikroverfilmung von Bibliotheksbeständen auch in unterschiedlichen Mentalitäten (Konservativismus der Europäer) und geographischen Bedingungen (kürzere Distanzen in Europa) und prognostizierte 1939, dass europäische Bibliotheken ,zweifellos eher Quellen als Benutzer von Mikrofilm bleiben würden." ${ }^{67}$ In Deutschland konnten die Bibliotheken auf die hervorragende Technik von „Zeiss Ikon“ und der „Photokopie GmbH“ zurückgreifen. Es existierten Pläne, die gesamte Fernleihe im Jahr 1940 durch ein zentralisiertes Mikrofilmlabor in Berlin zu ersetzen, ein Vor-

62 Raney, American Trends, 75-79

63 Microfilm Abstracts, in: F. I. D. Communicationes 8 (1941), 20-21; Publication of Doctoral Theses by Microfilm, 53-54. Power, University Microfilms, 21-28.

64 Atherton Seidell, The Utilization of Microfilms in Scientific Research, in: Science 89, 2298 (1939), 32-34; ders., Microfilm Copying of Scientific Literature, in: Science 89, 2306 (1939), 219-220.

65 Emile Leroy, Le development du microfilm en france, in: F. I. D. Communicationes 6 (1939), 48-50; P. Poindron, Le microfilm en France, in: F. I. D. Communicationes 6 (1939), 83-85; L. De Saint Rat, Microphotocopies et Microtheques, in: I. I. D. Communicationes 4 (1937) H. 1, 21-24. 66 George A. Schwegmann, The Photoduplications Service of the Library of Congress, in: Journal of Documentary Reproduction 2, (1939) H. 3, 176-179.

67 Eugene B. Power, Microfilm in Europe, in: Journal of Documentary Reproduction 2 (1939) H. 4,258 . 
haben das angesichts der Kriegsentwicklung nie verwirklicht wurde. ${ }^{68}$ Die von L. Fritz Gruber 1940 gegründete Firma „Mikrozeitung“ stellte im Auftrag von Verlegern Kleinbildarchive von Tageszeitungen her. ${ }^{69}$ Ein wichtiges Anwendungsgebiet des Mikrofilms zu wissenschaftlichen Zwecken im nationalsozialistischen Deutschland war die Verfilmung von Kirchenbüchern des Deutschen Reiches im Auftrag der Reichsstelle für Sippenforschung. Anders als in den USA, wo eine fehlende Standardisierung, d.h. eine grosse Varietät an Leseapparaten und Formaten dominierte, welche die Austauschbarkeit der Medien massiv beeinträchtigte, beschäftigte sich der Ausschuss für photographische Vervielfältigungsverfahren beim Deutschen Normenausschuss bereits zu Beginn der 1940er Jahre mit der Normierung der Filme gemäss Deutscher Industrienorm (DIN). ${ }^{70}$

Mikrofilme stellten in mehrfacher Hinsicht tradierte Praktiken im Umgang mit der Publikation, Lagerung und Konservierung von Druckschriften in Frage. In diesem Zusammenhang sind die Befunde der Studie des Joint Committee on Material for Research aufschlussreich, die 1929 vom Social Science Research Council und anderen wissenschaftspolitischen Gremien in den USA in Auftrag gegeben wurde. Die Studie analysierte Drucktechniken, Bücher, Papier und Bibliotheken in Parametern der Effizienz. Effizienzverluste wurden an den unterschiedlichsten Orten eruiert: bei Übergrössen, Schriftgrössen und insbesondere den Kleinauflagen. Auf das massgeschneiderte Lektüreformat, das in Dokumentalistenkreisen bereits nach der Jahrhundertwende im Gespräch war, folgte jetzt die Idee der massgeschneiderten Vervielfältigungstechnik. Die Diffusion von Wissen sollte mittels Kostenkalkulation optimiert werden, wobei die Wahl der Vervielfältigungstechnik von der Auflagenhöhe abhängig gemacht werden sollte. ${ }^{71}$ Papier geriet als Konservierungsmedium in Verruf. Der Bericht empfahl, Zeitungen aus den Jahren 1875 bis 1910 wegen schlechter Papierqualitäten auf Mikrofilm zu übertragen und dabei „das Original verschwinden zu lassen“. ${ }^{72}$ Die Studie kam zum Schluss, dass man am Ende der Gutenberg Ära angelangt sei. Angesichts alternativer Vervielfältigungsmedien wie Mikrofilm und den damit verbundenen Möglichkeiten des ,publish on demand" seien die Arbeitsteilung zwischen Verlag und Bibliothek und die Unterscheidung zwischen Druck und Manuskript hinfällig geworden. Damit befinde man sich erstaunlicherweise in einer ähnlichen Situation wie vor dem Buchdruck: „If a monastery maintained a criptorium, and if its policy was to accede to requests for copies of its books, its whole library was, in a sense, ,published material'. Precisely

68 Ebd., 254-263; Walter Schürmeyer, Die Fortschritte der Filmfotokopie, in: F. I. D. Communicationes 6 (1939), 27-30. Walter Schürmeyer, Der Stand der Photomikrographie in Deutschland, in: F. I. D. Communicationes 6 (1939), 81-82.

69 Arpad von Biehler, Handbuch der Photokopie, Halle 1948, 178.

70 Felix Palic, Über die technischen Grundlagen des Bibliofilms, in: F. I. D. Communicationes 9 (1942), 33-38; Dokumentation und Arbeitstechnik. Zwanglose Mitteilungen des Fachnormenausschusses für Bibliotheks-, Buch- und Zeitschriftenwesen, Berlin 1940-1942.

71 Binkley, Manual, 183.

72 ,letting the original dissappear“, Ebd., 106. Die grossangelegten Kopierprojekte von Zeitungen in amerikanischen Bibliotheken liefen auf eine Zerstörung von Drucksachen hinaus. Vgl. dazu: Nicholson Baker, Double Fold. Libraries and the Assault on Paper, New York 2001. 
this situation returns to the degree that microcopy becomes generally available." 73 Nach Gutenberg ist vor Gutenberg: Die Analogie der modernen Verlagsbibliothek, welche die Funktion des Magazins, der Vervielfältigungswerkstatt und des Verteilzentrums vereint, mit der mittelalterlichen Bibliothek als einer Monopolinstitution über Wissen und Vervielfältigung ist auf den ersten Blick irritierend. Doch sie ist im Endeffekt gegen die Verlage gerichtet und zeugt von der Vision der Wissenschaftsrationalisierer der 1930er Jahre, Bibliotheken und wissenschaftliche Institutionen mittels moderner Technik als autonome Instanzen in der Gesellschaft zu positionieren und dabei das Potential neuer Medien für den Umbau tradierter Institutionen zu nutzen. Mehr als zwei Jahrzehnte später im Jahr 1962 veröffentlichte der Vordenker der Medienwissenschaft Marshall McLuhan in „The Gutenberg Galaxy“ einen breit rezipierten Nachruf auf das Buchdruckzeitalter. Er entwarf die These einer neuen ,elektrischen Galaxis“, welche bereits tief in die Gutenberg Galaxis eingedrungen sei, und deshalb zu beträchtlichen Kollisionen und zur Erosion vertrauter Institutionen geführt habe. ${ }^{74}$ Der Bericht des Joint Committee on Materials for Research ist deshalb historisch so bedeutsam, weil er die kulturellen Konsequenzen der neuen Vervielfältigungstechniken in aller Schärfe erstmals auf den Punkt brachte: Die Auflösung der Unterscheidung zwischen Publizieren und Sammeln, das Verschwinden der Bedeutung des Buchdrucks, was in letzter Konsequenz auf das Verschwindenlassen von Druckerzeugnissen hinauslief und der Aufstieg neuer Kommunikationskanäle für die wissenschaftliche Praxis (,That which is printed is only a fraction of that which is written and communicated" ${ }^{\text {"75 }}$ ). Er ist zudem eine interessante Quelle für die zeitgenössische Deutung von Praktiken in Wissenschaft und Bibliotheken. So wie in der betrieblichen Rationalisierungsbewegung die Ersetzung der menschlichen Arbeitskraft durch Maschinen gefordert wurde, diskreditierte der Bericht Editionsprojekte, die obligatorische Wegstrecke fast aller Geisteswissenschaftlervita, als physische und damit unwissenschaftliche Arbeit. Die Forscher hätten die physische Arbeit des Abschreibens mit einem intellektuellen Ergebnis verwechselt. ${ }^{76}$ Auch die Metaphern für Bibliotheken hatten sich in den 1930er Jahren gewandelt: Die Sprache der Elektrizität hielt Einzug in die Welt der Bibliotheken und Fliessmetaphern verdrängten das Lagerhaus als Chiffren der Bibliothek: „To librarians and archivists they present the issue of whether libraries and archive depositories are to be operated as storehouse of property or power houses of cultural energy. “77

Trotz des Redens über den Fluss von Dokumenten ${ }^{78}$ und der damit einhergehenden Vorstellung der Diffusion von Wissensressourcen über grosse Distanzen hinweg standen der Mikrofilmtechnik massive Anwendungsprobleme entgegen. Das Lesen der Dokumente auf Papier oder mittels Projektion gestaltete sich mangels ge-

73 Binkley, Manual, 160.

74 Marshall McLuhan, The Gutenberg Galaxy. The Making of Typographic Man, Toronto 1962.

75 Binkley, Manual, 197.

76 Ebd.

77 Robert C. Binkley, Techniques and Policies of Documentary Reproduction, in: F. I. D. Communicationes 6 (1939), 14.

78 Raney, American Trends, 79. 
eigneter Lesegeräte sehr schwierig und die eingeübten Kulturtechniken des Lesens von Druckschriften waren nur beschränkt übertragbar: Die Augen ermüdeten schnell und konnten sich nicht mehr wie gewohnt bewegen. ${ }^{79}$ Zudem stellte sich das Problem der fehlenden Standardisierung der Formate. Obwohl amerikanische Stiftungen französischen Bibliotheken Apparate und technisches „Know-how“ zur Verfügung gestellt haben, mussten sie resigniert feststellen, dass in Frankreich die Filme in Streifen geschnitten, in Umschläge verpackt und wie Bücher gelagert (statt gerollt) wurden. Die Library of Congress Mission, die deutschen wissenschaftlichen Bibliotheken nach dem Zweiten Weltkrieg die Kriegsjahrgänge amerikanischer Zeitungen als Kleinbildfilm zur Verfügung gestellt hat, konnte angesichts des Papiermangels und fehlender geeigneter Lesegeräte „nicht recht ausgenutzt“ werden, wie ein deutscher Bibliothekar 1948 trocken bemerkte. ${ }^{80}$ In den USA hatte es zu Beginn der 1940er Jahre bereits Stimmen gegeben, welche den Mikrofilm durch die Verwendung von Planfilm weg von den Konventionen des Films und näher an die Tradition des Buches bringen wollten. ${ }^{81}$ Die Formatfrage „Mikrofiche oder Filmrollen?" tendierte in Europa gleich nach dem Krieg eindeutig zu Gunsten der Mikrofichen entschieden zu werden, weil diese eine konstruktive Vereinfachung der Lesegeräte ermöglichten ${ }^{82}$ und auch weil sie sich in die in den Bibliotheken gebräuchlichen Kartothekkästen einordnen liessen. ${ }^{83}$

Es kostete schliesslich so viel Zeit in den endlosen Filmrollen einzelne Artikel zu suchen, dass die Ersparnis an Raum „unwesentlich“ wurde. ${ }^{84}$ Der amerikanische Wissenschaftspolitiker Vannevar Bush erkannte die Beschränktheit der Miniaturisierung und des Mikrofilms als Speichermedium im Essay „As We May Think“ zu Kriegsende bereits im Juli 1945: „More compression, of course is not enough; (...) one need not only to make and store a record but also be able to consult it." 85 Er skizzierte die Vision eines neues Wissensspeichers, zwar unter Verwendung des alten Mediums Mikrofilm, aber „Memex“, wie er den individuell gestalteten Zukunftsspeicher taufte, sollte die bestehenden Ordnungs- und Findmittel durch die Schaffung eines Wegnetzes aus assoziativen Indexen übertreffen.

79 M.F. Donker Duyvis, La photocopie micrographique sur papier, in: I. I. D. Communicationes 2 (1935) H. 2, 16-23.

80 Wilhelm Hinsch, Grundlagen und Möglichkeiten des Mikrobuches, in: Centralblatt für Bibliothekswesen 62 (1948), 282.

81 James Reyniers/P. C. Trexler/R. F. Ervin, The Use of Flat Film for Microcopy, in: Journal of Documentary Reproduction 4 (1941) H. 1, 3.

82 L. De Saint Rat, Le microfilm dans la vie pratique, in: F. I. D. Communicationes 13 (1946), C55-C60; Hinsch, Grundlagen, 278-291; Heinz Lübeck, Grundsätzliche Fragen des Mikrobuches, in: Centralblatt für Bibliothekswesen 63 (1949), 17-29.

83 Zur Geschichte des Karteikastens: Markus Krajewski, Zettelwirtschaft. Die Geburt der Kartei aus dem Geiste der Bibliothek, Berlin 2002.

84 K. Hijmans, Fotokopie oder Mikrokopie für die Dokumentation, in: I. I. D. Communicationes 4 (1937) H. 1, 7-9.

85 Vannevar Bush, As We May Think, in: Athlantic Monthly 1945, http://www.theatlantic.com/ doc/194507/bush (02.12.2007). Versuche mit Suchmaschinen für Mikrofilme gab es schon zu Beginn der 1930er Jahre durch Emanuel Goldberg angesichts des „Registrierproblems in der Photographie“, vgl. Michael Keeble Buckland, Emanuel Goldberg and His Knowledge Machine. Information, Invention, and Political Forces, Westport/Conn. 2006. 


\section{Vom Photoreglement zum Gentlemen's Agreement}

Bis zum Jahr 1930 wurden die Photokopierer und die Mikrofilmapparate weder in Europa noch den USA im Zusammenhang mit Urheberrecht bzw. Copyright diskutiert. Das bedeutet nicht bloss, dass bis zu diesem Zeitpunkt nie vor Gericht auf Urheberrechtsverletzung geklagt wurde, sondern auch, dass die Frage ob Bibliotheken kopieren dürfen, bis 1930 schlicht nicht gestellt wurde, weder im Kontext der Bibliotheken und der Reproduktionstechnik noch in der Rechtswissenschaft. Mitte des 19. Jahrhunderts war die nationalstaatliche Regelung des Autorrechtes bereits vollzogen (in den USA im Jahre 1790, in Frankreich 1793, in Preussen 1837 bzw. dann 1870 im Deutschen Reich). Diese Gesetze waren das Resultat eines Zusammenspiels ästhetischer und rechtlicher Diskurse im 18. Jahrhundert, die den „Autor“" ins Zentrum einer neuen Gesetzgebung stellten und ihm während einer limitierten Zeitfrist ökonomische und teilweise auch moralische Rechte auf sein Werk gewährten. ${ }^{86}$ Gleichzeitig gab es seit den 1840er Jahren Bemühungen um eine Internationalisierung nationaler Normen, die zunächst in bilaterale Abkommen und dann 1886 zur Unterzeichnung einer internationalen Konvention, der „Berner Übereinkunft zum Schutze von Werken der Literatur und Kunst" führten.

Viele Staaten hatten ihre Gesetze kurz nach 1900 revidiert (Deutschland 1907, USA 1909, Grossbritannien 1911) und damit gerade mal die Photographie in das Rechtsgebäude integriert. Als in den 1930er Jahren die Photokopie und der Mikrofilm als Arbeitsressource für Wissenschaft und Dokumentation zur Anwendung kam und dabei nicht mehr bloss Handschriften und alte Drucke, sondern auch aktuellste Zeitschriftenliteratur kopiert wurde, erhoben die Urheberrechtsfachkreise, Bibliotheken und die Dokumentationsbewegung die Kopie zur Rechtsangelegenheit. Die Urheberrechtsfrage der Photokopie wurde zu diesem Zeitpunkt nirgends so intensiv debattiert wie in Deutschland. 1930 erschien das erste einer Reihe von Rechtsgutachten zu den Bibliothekskopien. ${ }^{87}$ Grundsätzlich verwiesen alle Gutachten auf das Urheberrecht, wonach die Vervielfältigung von geschützten Werken nicht erlaubt sei. Doch hinsichtlich der Beurteilung der Bibliothekskopien waren die Ansichten gespalten: Während die einen jede Kopie von der Einwilligung des Urheberrechtsinhabers abhängig machen wollten, plädierten die anderen für eine

86 Zur wechselseitigen Beziehung von neuen Autorkonzepten und der Genese eines Urheberrechts im 18. Jahrhundert: Gerhard Plumpe, Eigentum - Eigentümlichkeit. Über den Zusammenhang ästhetischer und juristischer Begriffe im 18. Jahrhundert, in: Archiv für Begriffsgeschichte 23 (1979), 175-196.

87 Max Stois, Urheberrechtliche Fragen aus der Bibliothekspraxis, in: Centralblatt für Bibliothekswesen 47 (1930), 629-642; Alexander Elster, Zur Photokopie-Frage, in: Gewerblicher Rechtsschutz und Urheberrecht 36, 9 (1931), 952-955; Kurt Alexander, Das Photokopierverfahren im Licht von Wissenschaft und Praxis: zugleich eine Erwiderung auf Elsters: „Zur Photokopierfrage“, in: Gewerblicher Rechtsschutz und Urheberrecht 36, 33 (1931), 1117-1124; Friedrich Labes, Die Rechtsgrundlage der im Auftrage von Bibliotheksbenutzern hergestellten Kopien, in: Centralblatt für Bibliothekswesen 49 (1932), 240-249; Willy Hoffmann, Das Photokopierverfahren, in: UFITA 5 (1932), 62-65; Walter Schürmeyer, Die Photographie im Dienste der bibliothekarischen Arbeit, in: Centralblatt für Bibliothekswesen 50 (1933), 580-583; Treplin, Bibliotheksrecht, 597-634; Schreiber, Pflicht, 441-463. 
Ausnahmegenehmigung, wenn die Kopie anstelle einer Ausleihe, zum persönlichen Gebrauch und ohne gewerbliche Interessen hergestellt werde. Bibliotheksnahe Gutachter betonten, dass die mechanische Kopie das manuelle Abschreiben sowie die Ausleihe und nicht den Erwerb eines Buches ersetze und verwiesen auf die im Gesetz von 1907 verankerte Beschränkung des Urheberrechts im Zusammenhang mit „persönlichem Gebrauch“. Die Rechtssprechung berief sich im einzigen Urteil zur Bibliothekskopie in den 1930er Jahren auf die Gewerbsmässigkeit als Ausschlusskriterium für die Rechtmässigkeit des Kopierens, als das Landgericht Leipzig im Dezember 1932 die Photocopie GmbH zu Schadensersatz wegen Urheberrechtsverletzung durch Photokopien verurteilte. Interessant ist bei diesem Urteil, dass die den Bibliotheken gewährten Rechte kommerzielle Tätigkeiten dezidiert ausschlossen. Bibliotheken wurden als interessenfreie Zone ausserhalb des Wirtschaftslebens konzeptualisiert.

Das Institut International de Documentation (IID) lancierte die Frage nach dem rechtlichen Rahmen der Bibliothekskopie im Jahr 1932: Sie sei zu bedeutend für die Zukunft der Bibliotheken, als dass sie den Aktivitäten von Autoren- oder Verlagsvertretern überlassen werden könne. ${ }^{88}$ In Analogie zum Autorrecht wurden ein „Leserrecht“ und eine Interessenorganisation der Leserinteressen gefordert. Man dachte an jene Leser, die lesen, um Neues zu produzieren. Zum ersten Mal wurde dabei die Idee entwickelt, den Prozess des Photokopierens nach Vorbild der musikalischen Verwertergesellschaften an eine Abgabe zu koppeln, und damit Autoren und Verleger finanziell an der Kopie zu beteiligen. Damit sollte die Photokopie ohne zeitraubende Verfahren zur Klärung der Zustimmung von Autoren und befreit von Rechtsunsicherheit im grossen Stil für die Dokumentation genutzt werden können. ${ }^{89}$ Der Fluss von Kopien sollte durch einen Geldstrom rechtlich ausbalanciert werden: Statt einer Kopie an die national zentralisierte Instanz der Bibliothek (wie beim ,depot legal“) dachte man nun an einen Geldtransfer an eine zentralisierte internationale Instanz (das IID). Das dahinter stehende bürokratische Modell des Geldtransfers von einer internationalen Organisation über nationale Organisationen an einzelne Autoren wurde jedoch von Kommentatoren für undurchführbar erklärt: die pekuniäre Kompensation drohte angesichts des administrativen Aufwands vollständig zu versickern. ${ }^{90}$ Es ist kein Zufall, dass die Idee einer Kopierabgabe im Kontext der Dokumentation geboren wurde, die neben der Verwaltung und der Wissenschaft auch in kommerziellen Unternehmen tätig war und sich damit in einer Mischzone zwischen Bibliothek und Wirtschaft konstituierte.

In den USA wurde die Frage der Vervielfältigung von copyright-geschützten Werken zu Beginn der 1930er Jahre vom bereits erwähnten Joint Committee on

88 C.J. De Haan, Photocopie et droit d'auteur, in: I. I. D. Communicationes 1 (1934) H. 4, 25 40.

89 P. Bourgeois, La reproduction des documents en relation avec les droits d'auteur \& d'éditeur, in: Internationales Institut für Dokumentation (I. I. D.) (Hg.), Vorträge der 11. Konferenz, Bruxelles 1932, 38.

90 Luise von Schwartzkoppen, Die rechtliche Zulässigkeit der Photokopie im Rechte des Auslands und nach dem Entwurf zu einem neuen Urheberrechtgesetz, in: Zentralblatt für Bibliothekswesen 51 (1934) H. 6, 307. 
Materials for Research aufgegriffen. Das Ziel war Rechtsunsicherheit zu klären und die rechtlichen Grundlagen für das Kopieren im wissenschaftlichen Kontext zu schaffen. ${ }^{91}$ Ursprünglich verfolgte die Kommission die Idee, die im englischen Copyright Act von 1911 festgeschriebene und im amerikanischen Common Law etablierte „fair use“ Ausnahmeregelung für Forschung und Studium in einer zukünftigen revidierten Fassung des amerikanischen Copyright Act auch auf Ebene des Gesetzes zu fixieren. Doch die Verleger bekundeten in informellen Treffen, einer solchen Ausnahmenregelung nicht zustimmen zu können, weil sie dadurch das Rechtsgebäude des Copyrights in Gefahr sahen. Die informellen Treffen zwischen Bibliothekaren und Verlegern mündeten in ein „Gentlemen's Agreement“. Es war kein Vertrag, sondern eine schriftliche Erklärung, die von der Kommission, dem Joint Committee on Materials for Research, und der National Association of Book Publishers 1935 unterzeichnet wurde. Die Erklärung hielt fest, dass Bibliotheken für Forscher einzelne Photo- und Mikrokopien herstellen dürften, im Tausch gegen eine schriftliche Erklärung, dass die Kopie anstelle des Abschreibens oder einer Ausleihe und ausschliesslich zu Forschungszwecken erfolge. Die Form und der Inhalt der Erklärung spiegeln die Idee, dass der drohende Kopierkonflikt unter Geschäftspartnern am runden Tisch als Frage der Moral und Ehre geregelt werden könnte. Schreibakte wie die Unterschrift des Wissenschaftlers und das Anbringen von Warnungen auf den Kopien sollten das Recht symbolisch repräsentieren und auf seinen möglichen Bruch durch den Akt des Kopierens verweisen. Die Bibliotheken hofften dadurch die rechtliche Verantwortung für die Kopie an den Wissenschaftler zu delegieren und Klagen zu vermeiden. Gleichzeitig verschafften sie dem Copyright durch diese Praxis eine permanente Präsenz in der sich veralltäglichenden Kopierkultur. Die Kopie figurierte somit als Verbreitungsmedium des Copyrights.

Die Photokopie war zu diesem Zeitpunkt noch zu neu, um in die langwierigen nationalen Gesetzgebungsprozesse integriert zu werden und sie war zu speziell, als dass sie Aussicht hatte, im internationalen Recht Erwähnung zu finden, obwohl die Kopierpraktiken sich durch die internationale Verflechtung der Wissenschaft in einem internationalen Kontext abspielten. ${ }^{92}$ Auffällig ist die Konvergenz der Entwicklung in den USA und in Deutschland, die trotz unterschiedlicher politischer und rechtlicher Systeme dies- und jenseits des Atlantiks in eine Politik des „Laisser-faire" mündete. Robert Binkley, der Vorsitzende des Joint Committee on Materials for Research, sprach 1939 (zwanzig Jahre vor McLuhan) von neuen „Kommunikationskanälen“93 und plädierte dafür diese offenzuhalten - „toward a freer intercourse in the world community“94. In Deutschland dominierte zur Zeit des Na-

91 Binkley, Manual, 135-139. The Gentlemen's Agreement and the Problem of Copyright, in: The Journal of Documentary Reproduction 2 (1939) H. 1, 29-36.

92 Vgl. F. Ostertag, Die Photokopie im Lichte des Urheberrechts, in: Internationales Institut für Dokumentation (Institut International de Bibliographie) I. I. D. (Hg.), Rapports Conference de documentation Copenhague 1935, Bruxelles 1935, Os. 2.

93 ,channels of communications“, Binkley, Techniques, 12.

94 Binkley, Techniques, 15. 
tionalsozialismus die Rede vom „geistigen Leben des Volkes“95 sowie die Vorstellung, dass Bibliotheken als „Wechselstuben geistiger Werke“ Privilegien gegenüber dem Urheber zustünden. ${ }^{96}$ Bemerkenswert ist auch, dass niemand das Urheberrecht beziehungsweise das Copyright grundsätzlich in Frage stellte. Auch nicht die in Europa entstandene Dokumentationswissenschaft oder wissenschaftspolitische Expertengremien in den USA, obwohl diese die kulturellen Prämissen und die zentralen Instanzen des Buchdruckzeitalters zu diesem Zeitpunkt bereits zum Auslaufmodell erklärt hatten.

Unterdessen kündigte sich eine neue Technologie an: 1937 liess Chester Carlson, ein Patentanwalt mit Physikausbildung aus New York, ein Verfahren patentieren, das er „Elektrophotographie“ nannte und das auf dem Zusammenspiel von elektrostatischer Aufladung, lichtempfindlicher Fläche und einem Tonerpulver beruhte. ${ }^{97}$ Das Patent war kein Erfolg. Erst 1945 stieg das Batelle Memorial Institute in die Weiterentwickelung des Verfahrens ein und erst 1947 entschied sich Haloid, ein Hersteller von Photopapier, in die Technik zu investieren. Die amerikanischen Bibliotheken kamen Mitte der 1950er Jahre durch „Copyflo“, den Mikrofilmprinter von Haloid, erstmals in Kontakt mit einer vollautomatischen elektrostatischen Kopiermaschine. ${ }^{98}$ Nur Bibliotheken mit hohem Kopierbedarf konnten die Miete des riesigen Apparates amortisieren: „Once you have it, you must keep it busy or else you find that you are working for the Haloid Xerox“, 99 warnte der Leiter des Kopierservices der Columbia University 1959. Doch es war nun das erste Mal möglich, einzelne Kopien eines Buches zu einem Preis von ein paar Cents pro Seite zu reproduzieren. Gerade vergriffene Bücher wurden in den Bibliotheken auf Nachfrage mit dem Copyflo reproduziert. Die Firma University Microfilms entwickelte daraus einen neuen Geschäftszweig und betrieb auf Basis von Lizenzen mit Verlegern einen Nachdruckservice für „out of print“ Dokumente. Fast gleichzeitig mit der Marktreife der ersten Kopierapparate von Haloid brachten Konkurrenten kleinere Kopierer auf der Basis von weiteren neuen Vervielfältigungstechniken auf den Markt. Damit war zu Beginn der 1950er Jahre an der Schnittstelle zwischen der Photo- und Bürobedarfsindustrie eine Wirtschaftsbranche erstarkt, deren Ursprünge in die 1920er Jahr zurückgehen: die „Rapid Copy Industry“. Die neuen Verfahren waren allesamt schneller, billiger und weniger personalintensiv als die bisherigen Kopiertechniken. Die Firma Haloid, die sich inzwischen Haloid-Xerox nannte, präsentierte im Dezember 1959 den „Xerox 914“, jenes Modell, das bald zum neu-

\footnotetext{
95 Schreiber, Pflicht, 463.

96 Schreiber, Pflicht, 442.

97 Zur Wissenschafts- und Wirtschaftsgeschichte von Xerox: David Owen, Copies in Seconds. How a Lone Inventor and an Unknown Company Created the Biggest Communication Breakthrough Since Gutenberg - Chester Charlson and the Birth of the Xerox Machine, New YorkLondon 2004; Joseph Mort, Xerography: A Study in Innovation and Economic Competitiveness, in: Physics Today 47, April (1994), 32-38; Robert W. Gundlach, Retrospective on Xerography and Chester S. Carlson, in: Frederick Su (Hg.), Technology of Our Times: People and Innovation in Optics and Optoelectronics, Belligham-Washington 1990, 56-62.

98 Vgl. Hubbard W. Ballou, Developments in Copying Methods - 1958, in: Library Resources \& Technical Servises (LRTS) 3 (1959) H. 2, 86-97.

99 Ballou, Developments, 88.
} 
en Synonym für das Kopieren avancieren sollte. Erstmals konnte auf gewöhnlichem Papier statt photochemisch behandeltem Spezialpapier und ohne den Einsatz von Flüssigkeiten (wenn auch mit Lärm-, Wärme- und Geruchsemissionen) kopiert werden. Der Xerox 914 konnte für 95 Dollar im Monat gemietet werden. ${ }^{100}$ Bibliothekare äusserten sich euphorisch über die Kopien, welche die Originale teilweise an Qualität übertreffen würden, weil die Typographie in einem gleichmässigeren Schwarz erscheine als bei schwachen oder ungleichmässigen Drucken oder Typoskripten. ${ }^{101}$ Die Handhabung des Apparates war extrem einfach, sechs Kopien pro Minute konnten hergestellt werden. Der Unterhalt erwies sich als weit schwieriger, Papierstau war an der Tagesordnung. Die Berichte über den automatischen Xerox 914 sind gespickt mit Anekdoten über sein Nicht-Funktionieren. Die Preise von Haloid beliefen sich pro Kopie auf knapp fünf Cents bei einem Volumen von mindestens 2000 Kopien im Monat. Mit dem Xerox 914 seien in den Bibliotheken neue Kopiergelüste geweckt worden, meinte der Verantwortliche der Reproduktionsabteilung an der University of California in Los Angeles im Jahr 1964 rückblickend. ${ }^{102}$ Die Anzahl Kopien sei sprunghaft in die Höhe geschellt. Die Bibliotheken hätten alsbald die Öffnungszeiten des Kopierservices am Abend und an den Wochenenden erweitern und zusätzliche Apparate anschaffen müssen. Dass die Bibliotheken angesichts der grossen Zahl der Kopieraufträge ihre Organisation und Verwaltung anpassen mussten, spiegelt sich auch darin, dass Bibliotheken dazu übergingen ihren Kunden eine „Xerox Credit Card“ zu verkaufen: Die Karte im Wert von 10 \$ wurde bei jeder Kopie gelocht; damit ersparte sich die Bibliothek ein aufwändiges Buchhaltungssystem. ${ }^{103}$ Eines war klar: Durch die Automatisierung des Verfahrens, den Trend zur Selbstbedienung (Mikrofilmprinter waren bereits mit Münzapparaten versehen) und die Diffusion des Apparates an stark frequentierten Orten wie Bahnhöfen waren Aussagen darüber, wer was wie oft kopierte, unmöglich geworden. Bibliothekare verloren damit die Kontrolle über den Kopierprozess ihrer Bestände.

\section{Vom „Laisser-faire“ zur Grundsatzdebatte um Copyright}

Die Geschichtsschreibung verdankt den Rechtskonflikten viele Quellen. Im Falle der sich zu Beginn der 1950er Jahre (und nicht erst durch den Xerox 914) besonders in den USA erhitzenden urheberrechtlichen Konfliktzone um die Photokopie wurde viel Papier bedruckt (und bestimmt auch viel kopiert), wobei insbesondere zwei Studien für die Kulturgeschichte der Photokopie aufschlussreich sind: Eine

100 Rolland E. Stevens, Library Experiment with the Xerox 914 Copier, in: Library Resources \& Technical Servises (LRTS) 6 (1962) H. 1, 25-29; Helen Crawford, Notes of a Librarian on Contemplating Her Xerox Machine, in: Bulletin of the Medical Library Association 51 (1963) H. 3, 397-399.

101 Stevens, Library, 26.

102 William R. Hawken, Reprographic Technology: Present and Future, in: Lowell H. Hattery/ George P. Bush (Hg.), Reprography and Copyright Law, Washington D. C. 1964, 48.

103 Crawford, Notes, 398. 
erste quantitative Studie aus dem Jahr 1961 des Joint Library Committee on Fair Use in Photocopying befragte drei amerikanische Bibliotheken im Jahr 1959 zum Ausmass des Kopierens und den dabei betroffenen Beständen. ${ }^{104}$ Eine zweite qualitative Studie, die im Jahr 1962 von der National Science Foundation (NSF) im Zusammenhang mit Forderungen der Verleger nach Kopierabgaben in Auftrag gegeben wurde, interviewte Wissenschaftler, Verleger und Bibliothekare zum Kopierverhalten. ${ }^{105}$ Die Studien geben Aufschluss darüber, wie die neue Arbeitstechnik von Wissenschaftlern verwendet und wahrgenommen wurde und wie sie die Frage des Copyrights beurteilten. Zwischen 50\% (Princeton) und 92\% (New York Public Library) aller kopierten Texte stammten aus Zeitschriften, fast ausschliesslich aus Jahrgängen des 20. Jahrhunderts. Die Hälfte der Artikel stammte gar aus den letzten zehn Jahren, über 40\% waren im Ausland erschienen. Über 90\% der Wissenschaftler gaben an, Kopien nach Gebrauch aufzubewahren, und ebenfalls so viele Forscher berichteten, dass sie Kopien auch an andere Wissenschaftler weitergeben. Wissenschaftler betrachteten Photokopien ganz klar als Diffusionsmedium. Sie hatten kaum Kenntnisse über das Copyright und massen ihm auch keine besondere Bedeutung zu. „Piraterie“ oder der „Missbrauch“ von Forschungsresultaten durch Nichtzitieren, Falschzitieren oder die Verwendung von Forschungsresultaten durch kommerzielle Unternehmen beschäftigte die Forscher weitaus stärker. Zudem beklagten sie, durch die Kopie die Übersicht über ihre Leser zu verlieren: „You don't know who is interested in your work". ${ }^{106}$ Die von Bibliotheken auf Verlangen zur Verfügung gestellte Kopie machte das Bestellen von Reprints beim Autor hinfällig. Die persönliche Korrespondenz anlässlich des Bestellens und Versendens des Reprints entfiel durch die Kopie. Die Studie offenbarte damit einen Wandel sozialer Beziehungen und der Kommunikationspraxis durch die Kopie. Die Kommunikation durch persönliche Beziehungen wich teilweise einer Kommunikation durch ein technikvermitteltes Massenmedium. ${ }^{107}$ Sie manifestierte ausserdem ihr hohes Zirkulationspotential und den Multiplikatoreffekt der Kopie durch die Praxis des Tauschens von Kopien unter Wissenschaftlern. Eine Kopie schuf eine Kopie schuf eine Kopie etc.

Die Ergebnisse der NSF-Studie spiegelten das Wissenschaftsverständnis wider, das fünfzehn Jahre früher vom geistigen Vater der NSF skizziert worden war: Vannevar Bush, der Forschungskoordinator der Kriegswissenschaft in den USA, hatte im Sommer 1945 in seiner Skizze zur Gründung einer staatlich finanzierten Stiftung für die Grundlagenforschung (die spätere NSF) dem internationalen „Informationsfluss“ höchste Priorität zugewiesen. ${ }^{108}$ Grundlagenforschung diene der nationalen Wohlfahrt der USA und bedürfe eines „rapid flow of information“. „In-

104 Joint Libraries Committee on Fair Use in Photocopying, in: Bulletin of the Copyright Society of the U.S.A. 9 (1961) H. 1, 79-84.

105 Survey of Copyrighted Material Reproduction Practices in Scientific and Technical Fields, in: Bulletin of the Copyright Society of the U.S. A. 11 (1963) H. 2, 69-124.

106 Ebd., 84.

107 Niklas Luhmann, Die Realität der Massenmedien, Wiesbaden 2004 [Opladen ${ }^{1} 1995$ ].

108 Vannevar Bush, Science, the Endless Frontier. A Report to the President on a Program for Postwar Scientific Research, Washington D. C. 1960 [ $\left.{ }^{1} 1946\right], 22$. 
formationsfluss", ein Konzept aus dem Kontext der Kybernetik, avancierte in den USA nach dem Zweiten Weltkrieg zu einem wissenschaftlichen Schlüsselbegriff, der auch wissenschaftspolitisch in die Waagschale geworfen wurde. ${ }^{109}$ Die Siegermacht USA hatte nach Kriegsende den Anspruch im internationalen wissenschaftlichen und kulturellen Austausch eine führende Rolle zu übernehmen. ${ }^{110}$ Zudem sollte Wissenschaft nun im Kalten Krieg angesichts des Bedrohungsszenarios des Kommunismus wiederum als Waffe dienen. Freier Informationsfluss figurierte dabei auch als Gegenmodell zur Informations- und Vervielfältigungskontrolle des Feindes. ${ }^{111}$

Die Prämisse des schnellen Informationsflusses als Vehikel gesellschaftlicher Wohlfahrt erhielt zu Beginn der 1960er Jahre durch das neu entstehende Forschungsfeld der Szientometrie statistisch unterlegte Unterstützung: Der amerikanische Wissenschaftsforscher Derek de Solla Price führte in seiner Publikation „Little Science, Big Science“ nicht bloss das exponentielle Wachstum der „scientific community" und ihrer Zeitschriftenpublikationen in bestechenden Graphiken vor Augen, sondern auch die damit zusammenhängende sinkende Halbwertszeit von wissenschaftlichen ,papers“, gemessen an der sich rasant verkürzenden Zitationsphase einer Publikation. ${ }^{112}$ Dieser Befund bestärkte das Credo der Notwendigkeit einer Beschleunigung der Diffusionskanäle und damit auch des Mehrwerts der Kopien.

Seit den 1950er Jahren organisiert sich der Diskurs über die Bibliothekskopie anhand zweier Pole: Auf der einen Seite wird die Kopie als neues Diffusionsmedium in einer als Reputationsökonomie funktionierenden Wissenschaft modelliert, auf der anderen Seite als neues Verwertungsmedium von Urhebern in einer Geldökonomie stark gemacht. In Grossbritannien veröffentlichte die Royal Society 1950 eine Fair Copying Erklärung, wonach die Photokopie für wissenschaftliche und technische Zeitschriftenliteratur nur unter bestimmten Umständen als „fair use“ zulässig sei. Zudem befasste sich auch der British Board of Trade mit dem Thema und verfasste 1952 Empfehlungen für die Definierung von fair use bei einer zukünftigen Gesetzesrevision. ${ }^{113}$ Ein Kommentator stellte in der britischen Zeitschrift „Nature“ in Abrede, dass die Vorschläge auf Basis einer „geeigneten Einschätzung der Natur der wissenschaftlichen Kommunikation“ erstellt worden seien: „The

109 Vgl. dazu auch: Ronald R. Kline, Cybernetics. Management Science, and Technology Policy, in: Technology and Culture 47 (2006) H. 3, 513-535.

110 Vgl. dazu auch die 1946 durchgeführte Konferenz zur Rolle der amerikanischen Bibliotheken für internationalen wissenschaftlichen und kulturellen Austausch: American Library Association (Hg.), Conference on International Cultural, Educational, and Scientific Exchanges: Princeton University 1946. Preliminary Memoranda by Edwin Everitt Williamsa and Ruth Verrill Noble. Recommendations Adopted, Summary of Discussions, Chicago 1947.

111 In der DDR war die Herstellung von Druck- und Vervielfältigungserzeugnissen durch eine Verordnung aus dem Jahr 1959 genehmigungspflichtig: Institut für Verwaltungsorganisation und Bürotechnik Leipzig (Hg.), Vervielfältigen - aber wie?, Berlin 1965.

112 Derek John de Solla Price, Little Science, Big Science, New York-London 1963, 62-91.

113 Louis Charles Smith, The Copying of Literary Property in Library Collections. Reproduced from the Law Library Journal, August 1953 and August 1954 (Library of Congress Washington D. C.), o. O. $1954,8-9$. 
scientific man's financial return is often nil, or a merely nominal sum. His real profit is in the form of an increase in professional reputation. Indeed, his interest, like that of scientific progress, demands that his works should be circulated as widely and as freely as possible."114 Diese Kritik verweist auf einen „Wissenschaftsethos“, wie er vom Wissenschaftsforscher Robert Merton 1942 zum Zeitpunkt der massiven Interessengebundenheit der Wissenschaft im Zweiten Weltkrieg propagiert worden war. ${ }^{115}$ Ein Stützpfeiler des wissenschaftlichen Wertesystems, den Merton als „Kommunismus“ bezeichnete, schliesse es aus, dass ein Wissenschaftler ,geistiges Eigentum“ besitzen dürfe. Wertschätzung und Anerkennung seien seine Erträge. Obwohl der Patentkritiker Merton sich zum Copyright nicht explizit äusserte, liess er keinen Zweifel daran, dass das „Privateigentum“ eines kapitalistischen Wirtschaftssystems mit dem „Wissenschaftsethos“ unvereinbar sei. Publikationen seien der wahre Anreiz in der Wissenschaft, die durch „,vollständige und offene Kommunikation“ für Fortschritt bürgten. Die von Merton propagierte Ethik eines wissenschaftlichen „Kommunismus“ (die gegenwärtig im Konzept der „Commons“ eine Renaissance erlebt) findet sich ironischerweise gerade auch als wissenschaftlichen Leitbild zur Zeit des Kalten Krieges in den kapitalistischen USA. Doch die propagierte scharfe Trennung von Wissenschaft und Wirtschaft entsprach keinesfalls der Realität: Wissenschaft, Wirtschaft und Politik waren enger miteinander verwoben, als die Rede der reinen basalen Wissenschaft vorgab. Die Copyrightkonflikte entbrannten denn auch im Grenzgebiet von Wissenschaft und Wirtschaft - bei der wissenschaftlichen Fachzeitschrift, die gleichzeitig wissenschaftliches Verbreitungsmedium von Forschern und eine ökonomische Ertragsquelle von Verlagen ist.

Eine internationale Erhebung der UNESCO aus dem Jahre 1963 zeigte, dass die Bibliotheken inzwischen weltweit eine relativ liberale Kopierpraxis auf Basis von „fair use“, „Privatgebrauch“ oder „persönlichem Gebrauch“ pflegten. ${ }^{116}$ War das amerikanische Gentlemen's Agreement von 1935 ein Versuch gewesen, die Bibliothekskopie nicht durch Gerichtsprozesse zu gefährden, zeigte sich bald die Brüchigkeit dieses Versuchs der Rechtsetzung: Der Verhandlungspartner der Bibliothekare, die National Association of Books Publishers, existierte seit den 1940er Jahren nicht mehr. Zudem waren die Zeitschriftenverleger gar nie Mitglied dieser Vereinigung. ${ }^{117}$ Ein Bibliothekar kritisierte das Abkommen im Jahr 1962, weil die Bibliotheken damit vorschnell ein ihnen zustehendes Recht aus der Hand gegeben hätten. ${ }^{118}$ Die Erklärung habe die Bibliothek zu einem „Empfänger der Grosszü-

114 Leslie Wilson, Copyright and the Scientist, in: Nature 170, 4339 (1952), 1108-1109.

115 Robert K. Merton, The Normative Structure of Science [1942], 273-275, in: ders., The Sociology of Science. Theoretical and Empirical Investigations, Chicago-London 1973. Vgl. dazu auch: Adrian Johns, Intellectual Property and the Nature of Science, in: Cultural Studies 20 (2006) H. 2, 146-147.

116 Bibliothèque Nationale (Paris), Photographic Reproduction and Copyright, in: Copyright Bulletin XVI, 1 (1963), 25-68.

117 Lowell H. Hattery/George P. Bush, (Hg.), Reprography and Copyright Law, Washington D. C. 1964, 159-171.

118 Verner W. Clapp, Library Photocopying and Copyright: Recent Developments, in: Law Library Journal 55 (1962), 10-15. 
gigkeit des Copyrightinhabers" degradiert und nicht dazu verholfen, traditionelle Rechte der Bibliotheken in einem neuen technischen Umfeld zu verankern. Im Zuge der Aushandlungen um eine Gesetzesreform des Copyright Act aus dem Jahre 1909 veränderte sich der Diskurs um Copyright in den 1960er Jahren zunehmend von einer Diskussion um gesetzestechnische Details zu einer gesellschaftspolitischen Grundsatzdebatte über die Folgen der Technikentwicklung für Verlage, die Wissenschaften, die Bibliotheken und die gesellschaftliche Wohlfahrt sowie die Frage, welche Rolle der Gesetzgeber dabei einnehmen solle. Dabei drängten die Interessenvertreter der Copyrightinhaber in den USA bereits zu Beginn der 1960er Jahre darauf Copyrightinhaber durch Lizenzverträge oder Abgaben an der florierenden „Rapid Copy Industry“ mitverdienen zu lassen. Das Modell der Musikverwertung, das sich die American Society of Composers, Authors and Publishers (ASCAP) in den 1920er Jahren gegen das neue Medium Radio vor Gericht erstritten hatte, diente als Vision einer Diversifizierung des Verlagsgeschäfts angesichts neuer Distributionsmedien. ${ }^{119}$

1968 verklagte die Williams \& Wilkins Company aus Baltimore, ein Verlag für wissenschaftliche Fachzeitschriften, die National Library of Health, seit den 1930er Jahren ein Pionier eines umfassenden Kopier- und Mikrofilmservices für Forscher. „Williams \& Wilkins Company vs. The United States“ wurde mitten in den Verhandlungen für einen neuen Copyright Act zu Beginn der 1970er Jahre zum historischen Gerichtsfall über die Bibliothekskopie, der auch in Europa genau verfolgt wurde. ${ }^{120}$ Das Verbreitungsargument der Wissenschaft und das Verwertungsargument der Verleger, welche die ökonomische Basis der wissenschaftlichen Publikationen in Gefahr sahen und neu absichern wollten, standen sich schliesslich vor dem Supreme Court gegenüber. Das Gerichtsverfahren endete unentschieden: Das Nicht-Verdikt spiegelte das gesellschaftliche Patt zwischen den divergierenden Interessen zu Beginn der 1970er Jahre, die von Forderungen nach einer massiven Erweiterung von Copyrights bis zu einer erstmals seit dem 19. Jahrhundert wieder aufflammenden Skepsis gegenüber den Copyrights reichten. ${ }^{121}$

119 Vgl. dazu: Monika Dommann, Notieren, Aufzeichnen, Vervielfältigen: Medientechnische Umbrüche von Musik im Urheberrecht, in: Ralf Adelmann/Jan Hesse/Judith Keilbach/Markus Stauff/Mathias Thiele (Hg.), Ökonomie des Medialen. Tausch, Wert und Zirkulation in den Medien- und Kulturwissenschaften, Bielefeld 2006, 149-165.

120 Williams \& Wilkins v. United States, U. S. Supreme Court, Records and Briefs, 420 US 376, 1974.

121 Zwei skeptische Kommentare von Copyrightjuristen: Benjamin Kaplan, An Unhurried View of Copyright, New York-London 1967; Stephen Breyer, The Uneasy Case for Copyright: A Study of Copyright in Books, Photocopies, and Computer Programs, in: Harvard Law Review 84 (1970) H. 2, 281-351. 


\section{Schluss: Kopieren als Tauschgeschäft und kulturelle Aneignung}

Die Vervielfältigung von Bibliotheksdokumenten ist so alt wie die Geschichte der Bibliotheken. Doch mit der Photographie stand seit Mitte des 19. Jahrhunderts eine Technik zur Verfügung, die es ermöglichte, Bibliotheksbestände aus ihrem geographischen, örtlichen und auch materiellen Kontext zu lösen. Damit war die Idee einer zentralen Weltbibliothek von Kopien, wie sie vom amerikanischen Literaturwissenschaftler Mills Gayley um 1900 entworfen wurde, genauso denkbar wie die Idee eines neuen Textmediums auf Mikrofilm als rationalisierte Lektüretechnik in einer arbeitsteiligen Wissenschaft, die kurze Zeit später im Kontext der Dokumentationswissenschaft erstmals formuliert wurde. Die Kopie wurde als Mechanisierung und Rationalisierung wissenschaftlicher Praxis interpretiert und propagiert: die Ersetzung der körperlichen Arbeit des Abschreibens durch Maschinen. Damit einher ging eine neue Zeitökonomie, eine Rationalisierung des Lesens und eine Delegierung von Erschliessungsarbeit an internationale bibliothekarische Infrastrukturen. Mikrokopien ermöglichten eine Miniaturisierung, Entmaterialisierung und Vernichtung von Bibliotheksbeständen und eine Ersetzung von Papier durch Kunststoff. Angesichts der Dekontextualisierung und Fragmentierung der Dokumente durch den Kopierprozess entscheidet deren Erschliessung durch Metadaten über den Gebrauchswert der Kopien. Das galt für die ersten Prismenaufnahmen genauso wie für die aktuellen Scanprojekte der Google Library, die unlängst in einem Blog der American Historical Association in dieser Hinsicht kritisiert wurden. ${ }^{122}$

Unterstützt durch kapitalkräftige Stiftungen nutzten amerikanische Bibliotheken die Kopiertechniken seit den 1930er Jahren intensiv, um der neuen Welt die Bibliotheksbestände Europas einzuverleiben. Das Projekt eines wissenschaftlichen und wirtschaftlichen „Take-offs“ der USA war begleitet von einem neuen Selbstbild der Bibliothek als Verteilzentrum von Informationen. Die Rede vom freien Informationsfluss war ein wichtiges wissenschaftspolitisches Argument, das den Wissenschaften im Kalten Krieg eine erstarkte gesellschaftliche Position und den Bibliothekskopien den Sonderstatus eines Trägers des Informationsflusses sicherte und in einem gewissen Ausmass vor Copyrightansprüchen schützte. Mit der Entstehung von Vervielfältigungsalternativen zum Buchdruck in den 1930er Jahren kam die grundsätzliche Frage auf, welche Funktion eine Bibliothek in der Welt nach Gutenberg einnehmen soll, welche Rechte ihr dabei zustehen sollen und ob dabei Gegenrechte eingefordert werden dürfen. Das Kopieren in Bibliotheken organisiert sich seit den 1930er Jahren in einer Mischzone zwischen Wissenschaft und Wirtschaft. Die Frage, wer zu welchen Bedingungen kopieren darf, ist dabei deshalb so konflikthaft, weil sich divergierende Anreizmodelle - Reputation und Geld - mischen.

Grosse Kopierprojekte sind immer auch Tauschgeschäfte: Bei den amerikanischen Mikrofilmprojekten in europäischen Bibliotheken in den 1930er Jahren wur-

122 Robert B. Townsend, Google Books: What's Not to Like?, AHA Today American Historical Association, April 30, 2007, http://blog.historians.org/articles/204/google-books-whats-not-tolike (02.12.2007). 
den Kopierrechte gegen Apparate und Sicherungskopien getauscht. Bei den aktuellen Digitalisierungsprojekten, über deren „terms of trade“ zurzeit unter Interessenvertretern intensiv verhandelt wird, könnte neben Geld und Werbeflächen auch Sichtbarkeit im Internet zum Objekt eines Tausches werden. Die digitale Bibliothek von morgen wird wohl Bibliothek, Verkaufsportal und Suchmaschine in einem sein. Schliesslich wurden umfassende Kopierprojekte seit Mills Gayleys Vorschlägen für eine zentrale Kopier- und Verteilinstanz von Faksimiles immer wieder von Akteuren in den USA vorangetrieben, während Europa verzögert, skeptisch oder gar ablehnend auf diese amerikanischen Kopieroffensiven reagierte. ${ }^{123}$ Kopieren ist immer auch mit der Aneignung anderer Kulturen verbunden und wird dementsprechend als Angriff auf die eigene Kultur kritisiert.

123 Jean-Noël Jeanneney, Googles Herausforderung. Für eine europäische Bibliothek, Berlin 2006 [Paris ${ }^{12005] .}$ 